Pacific Journal of Mathematics

ON THE NONEQUIVALENCE OF CONSERVATIVE
HAUSDORFF METHODS AND HAUSDORFF MOMENT

John R. EDWARDS AND STANLEy G. WAYMENT 


\title{
ON THE NONEQUIVALENCE OF CONSERVATIVE HAUSDORFF METHODS AND HAUSDORFF MOMENT SEQUENCES
}

\author{
J. R. EDWARDS AND S. G. WAYMEnT
}

In this paper we give a counter example to the theorem: A Hausdorff method is convergence preserving if and only if it is generated by a moment sequence as stated in "vectorvalued summability methods on a linear normed space" by $L$. C. Kurtz and D. H. Tucker, Proc. Amer. Math. Soc. 16 (1965) 419-428.

New results are also obtained which extend those known on the equivalence of the generalized Hausdorff moment problem with a generalized Riesz Representation Theorem, and a class of normed spaces is given in which the above mentioned does hold. The key tool in establishing these is the $v$-integral.

1. Introduction. In [4] Kurtz and Tucker consider summability methods in the setting of linear normed spaces. In that paper they establish an equivalence between a form of the Hausdorff moment problem and an integral representation theorem (Tucker [8]). In this paper we give a stronger formulation of the Hausdorff moment problem and establish its equivalence to the $v$-integral representation theorem in [1] in the setting of convex spaces. Also in [4] the authors claim to show that a Hausdorff method is regular if and only if it is generated by a moment sequence. However, the proof of sufficiency establishes only that a Hausdorff method generated by a moment sequence is weakly convergence preserving. Goodrich [3] and Remanujan [6] have shown, independenty, in the setting of convex spaces that a Hausdorff method is weakly convergence preserving if and only if it is generated by a weak moment sequence. In $\S 4$ of this paper we give an example which shows that in general it is not the case that a Hausdorff method generated by a moment sequence is convergence preserving. The remainder of the paper is devoted to obtaining sufficient conditions for a Hausdorff method to be convergence preserving, and we conclude by defining a class of normed spaces in which being generated by a moment sequence is both necessary and sufficient for a Hausdorff method to be convergence preserving.

In the first three sections of this paper, $X$ will denote an $F$-space and $Y$ a convex space unless otherwise stated. $L[X, Y]$ will denote the collection of continuous linear operators from $X$ into $Y$, and $C$ 
denotes the space of continuous functions from $[0,1]$ into $X$ with the topology of uniform convergence.

We shall use the analogues of the definitions given in [4] for the following: matrix summability method from $X$ to $Y$, convergence preserving method, regular relative to $L$, and Hausdorff summability method. It will be convenient to refer to the following form of the generalized Toeplitz Theorem, proofs of which can be found in [3], [4], [5].

Theorem 1.1. (Kurtz-Tucker, Ramanujan, Goodrich). A summability method $\Phi=\left(\phi_{n \nu}\right)$ is convergence preserving if and only if the following conditions are satisfied.

(RN) There is a pairing $(p, q)$ (Swong's notation [7]) and constants $k_{p-q}$ such that for each bounded sequence $\left\{x_{n}\right\} \subset X, q\left(\sum_{\nu=0}^{\infty} \dot{\phi}_{n \nu}\left(x_{\nu}\right)\right)$ $\leqq k_{p-q} \sup p\left(x_{\nu}\right)$.

(RS) For each $x \in X$ the sequence $\left\{\sum_{\nu=0}^{\infty} \phi_{n \nu}(x)\right\}_{n=0}^{\infty}$ is Cauchy.

(C) For each $x \in X$ and for fixed $\nu$ the sequence $\left\{\dot{\phi}_{n 2}(x)\right\}_{n=1}^{\infty}$ is Cauchy.

Furthermore a summability method $\Phi$ is regular relative to $L$ if and only if $(\mathrm{RN})$ and the following conditions hold.

$\left(\mathrm{RS}_{1}\right) \quad$ For each $x \in X, \lim _{n} \sum_{\nu=0}^{\infty} \dot{\phi}_{n \nu}(x)=L(x)$

$\left(C_{o}\right) \quad$ For each $x \in X, \lim _{n} \phi_{n \nu}(x)=\theta_{x}$.

We use the concept of moment sequence defined by Kurtz and Tucker, i.e., a moment sequence is a sequence of transformations $\left\{\mu_{n}\right\} \subset L[X, Y]$ such that the Hausdorff method generated by $\left\{\mu_{n}\right\}$ satisfies (RN). We emphasize that the above definition of moment is in general a stronger condition than Ramanujan's weak moment sequence and also that the convergence in 1.1 occurs in the topology of $Y$ and not in the weak topology of $Y$.

2. The $v$-integral. The purpose of this section of the paper is to present the basic properties of the $v$-integral as given in [2]. A set function $K$ on the half-open intervals $\mathscr{F}=\{(a, b] \subset(0,1]\}$ with values in $L[X, Y]$ is said to be convex with respect to length or more briefly convex if $\sum_{i=1}^{n} \lambda_{i} K\left(I_{i}\right)=K(I)$ for each $I \in \mathscr{J}$ and disjoint partition $\left\{I_{i}\right\}$ of $I$ over $\mathscr{F}$, where $\lambda_{i}$ is the ratio of the length of $I_{i}$ to that of $I$. If $K$ additionally satisfies the property that there is a pairing $(p, q)$ and constants $W K_{p-q}$ such that each finite disjoint collection $\left\{I_{i}\right\}$ in $\mathscr{F}$ and corresponding collection $\left\{x_{i}\right\}$ in $X$ implies $q\left(\Sigma\left[k\left(I_{i}\right)\right]\left(x_{i}\right)\right) \leqq W K_{p-q} \max _{j} p\left(\sum_{i=1}^{j} x_{i}\right)$, then $K$ is said to be of bounded $(p, q)$ convex variation. Suppose $f$ is a function from $[0,1]$ into $X$. Then to say that a convex set function $K$ is $v$-integrable with respect to $f$ means that $\lim _{\sigma} \sum_{\sigma} K\left(I_{i}\right)\left(f\left(x_{i+1}\right)-f\left(x_{i}\right)\right)$ exists in $\bar{Y}$ the completion of $Y$, where the limit is taken over the net of all partition $\sigma$ of $(0,1]$, 
and we denote this limit by $v \int_{0}^{1} K d f$.

3. A Moment Problem. In this section of the paper we formulate a moment problem analogous to that given in [4]. Our goal is to show the equivalence of the following two statements, $S_{2}$ being a formulation of the Hausdorff Moment Problem and $S_{1}$ being a generalized integral representation theorem. We shall assume $Y$ is complete.

Statement $S_{2}$. The sequence $\left\{\mu_{n}\right\} \subset L[X, Y]$ is a moment sequence if and only if there exist a $\psi \in L[X, Y]$ and a convex set function $K$ with values in $L[X, Y]$ which is of bounded $(p, q)$ convex variation such that for each $x \in X, \mu_{0}(x)=\psi(x)$ and $\mu_{n}(x)=v \int K d\left(t^{n} \cdot x\right)$ for $n$ $\neq 0$.

Statement $\mathrm{S}_{1}$. The linear transformation $T: C \rightarrow Y$ is continuous if and only if there is a $\psi \in L[X, Y]$ and a convex set function $K$ with values in $L[X, Y]$ which is of bounded $(p, q)$ convex variation such that $T(f)=\psi(f(0))+v \int_{0}^{1} K d f$.

Observe that sufficiency always holds in the case of $S_{1}[1]$. Sufficiency also holds in Statement $\mathbf{S}_{2}$. This can be seen by the following argument. Let $\left(\dot{\phi}_{n \nu}\right)$ denote the Hausdorff method generated by $\left\{\mu_{n}\right\}$. Since $K$ is of bounded $(p, q)$ convex variation, then by Theorem 3 in [1] there is a pairing $(p, q)$ with constants $W K_{p-q}$ and $|\psi|_{p-q}$ such that for $f \in C$

$$
q\left(v \int_{0}^{1} K d f\right) \leqq W K_{p-q} \sup p(f(t)-f(0))+|\psi|_{p-q} p(f(0)) .
$$

Therefore,

$$
\begin{aligned}
q\left(\Sigma \phi_{n \nu}\left(x_{\nu}\right)\right) & =q\left(v \int_{0}^{1} K d\left\{\sum_{\nu=0}^{n}\left(\begin{array}{l}
n \\
\nu
\end{array}\right) t^{\nu}(1-t)^{n-\nu} x_{\nu}\right\}\right) \\
& \leqq W K_{p-q} \sup p\left(\sum_{\nu=1}^{n}\left(\begin{array}{l}
n \\
\nu
\end{array}\right) t^{\nu}(1-t)^{n-\nu} x_{\nu}\right)+|\psi|_{p-q} p\left(x_{0}\right) \\
& \leqq W K_{p-q} \max p\left(x_{\nu}\right)+|\psi|_{p-q} p\left(x_{0}\right) \\
& \leqq\left(\left|W K_{p-q}\right|+|\psi|_{p-q}\right) \max p\left(x_{\nu}\right),
\end{aligned}
$$

and, hence, $\left\{\mu_{n}\right\}$ is a moment sequence.

REMARK 3.1. In the formulation of Statements $S_{1}$ and $S_{2}$ in [4] sufficiency does not hold. This is because the integral representation theorem of Tucker requires that the function $K$ which is of bounded 
semi-variation must take its values in $L\left[X, Y^{+}\right]$, the bounded linear operaters from $X$ into $Y^{+}$, the weak sequential extention of $Y$ [8]. The reason that we are able to give these sufficient conditions is that the convex set function $K$ given in the representation theorem in [1] does not suffer this complication.

\section{Theorem 3.2. The Statement $\mathrm{S}_{1}$ is equivalent to Statement $\mathbf{S}_{2}$.}

To prove this theorem we adapt the argument presented by Kurtz and Tucker in [4] to the present setting.

Proof of 3.2. Suppose that Statement $\mathbf{S}_{1}$ holds, and that $\left\{\mu_{n}\right\}$ is a moment sequence. Define the linear transformation $\mathscr{T}$ from the real polynomials into $L[X, Y]$ by $\mathscr{S}\left(\sum_{\nu=0}^{n} \alpha_{\nu} t^{\nu}\right)=\sum_{\nu=0}^{n} \alpha_{\nu} \mu_{\nu}$, and define $T$ from the polynomial with coefficients in $X$ by $T\left(\sum_{\nu=0}^{n} t^{\nu} x\right)=\sum_{y=0}^{n}[\mathscr{T}$ $\left.\left(t^{\nu}\right)\right](x)$. Our goal is to show that $T$ is continuous on the polynomials with coefficients in $X$ from which it follows it can be extended continuously to $C$. (That the polynomials are dense in $C$ follows from Lemma 3 [6]). Suppose that $q$ is a semi-norm on $Y$. Let $P$ denote an $X$-valued polynomial. Then,

$$
\begin{aligned}
T\left(B_{n} P\right)(t) & =\sum_{\nu=0}^{n}\left(\begin{array}{l}
n \\
\nu
\end{array}\right) T\left[t^{\nu}(1-t)^{n-\nu} P\left(\frac{\nu}{n}\right)\right] \\
& =\sum_{\nu=0}^{n}\left(\begin{array}{l}
n \\
\nu
\end{array}\right){ }^{n} \Delta^{-\nu} \mu_{\nu}\left(P\left(\frac{\nu}{n}\right)\right),
\end{aligned}
$$

where $B_{n} P$ denotes the $n^{\text {th }}$ Bernstein Polynomial of $P$.

Since $\left\{\mu_{n}\right\}$ is a moment sequence, then it follows that there is a seminorm $p$ on $X$ and a constant $k_{p-q}$ such that

$$
\begin{aligned}
q\left(T\left(B_{n} P\right)\right. & \leqq k_{p-q} \max _{\nu} p\left(P\left(\frac{\nu}{n}\right)\right) \\
& \leqq k_{p-q} \sup _{t} p(P(t)) .
\end{aligned}
$$

Lemma 10 in [4] implies that $P(t)=\left(B_{n} P\right)(t)-\sum_{\nu=1}^{k-1} P_{\nu}(t) / m^{\nu}$ where $P_{\nu}$ is a polynomial, with coefficients in $X$, which is independent of $m$, and $k$ is the degree of $P$. Therefore,

$$
\begin{aligned}
q(T(P)) & \leqq q\left(T\left(B_{n} P\right)\right)+\frac{1}{n} q\left(\sum_{\nu=1}^{k-1} P_{\nu}(t) / n^{\nu-1}\right) \\
& \leqq k_{p-q} \sup _{t} p(P(t))+\varepsilon
\end{aligned}
$$

for any $\varepsilon>0$ for large enough $n$. Therefore, $T$ is continuous on the polynomials hence on $C$, and Theorem 3 of [1] implies that Statement $\mathrm{S}_{2}$ holds. 
Suppose that Statement $\mathrm{S}_{2}$ holds and suppose $T: C \rightarrow Y$ is a continuous linear transformation. Then $T$ induces a continuous linear transformation from $C R$, the real-valued continuous functions from $[0,1]$ with sup-norm topology, to $L[X, Y]$ which is defined by $[\mathscr{G}(f)](x)=$ $F(f \cdot x)$. Define the sequence $\left\{\mu_{n}\right\}$ in $L[X, Y]$ by $\mu_{n}=\mathscr{F}\left(t^{n}\right)$ for each $n$. Observe that for a bounded sequence $\left\{x_{n}\right\}$,

$$
\begin{aligned}
\sum_{\nu=0}^{n}\left(\begin{array}{c}
n \\
\nu
\end{array}\right) \Delta^{n-\nu} \mu_{\nu}\left(x_{\nu}\right) & =\sum_{\nu=0}^{n}\left(\begin{array}{c}
n \\
\nu
\end{array}\right)\left[\mathscr{T}\left(t^{\nu}(1-t)^{n-\nu}\right)\right]\left(x_{\nu}\right) \\
& =T\left(\sum_{\nu=0}^{n}\left(\begin{array}{l}
n \\
\nu
\end{array}\right) t^{\nu}(1-t)^{n-\nu} x_{\nu}\right) .
\end{aligned}
$$

Since $T$ is continuous and since $\sum_{\nu=0}^{n}\left(\begin{array}{l}n \\ \nu\end{array}\right)\left(t^{\nu}(1-t)^{n-\nu}=1\right.$ for each $t$, it follows that the (RN) condition is satisfied for the Hausdorff method generated by $\left\{\mu_{n}\right\}$ and therefore $\left\{\mu_{n}\right\}$ is a moment sequence. Statement $\mathrm{S}_{2}$ implies that there exist $\psi \in L[X, Y]$ and a set function which is of bound $(p, q)$ convex variation such that $\mu_{0}(x)=\psi(x)$ and $\mu_{n}(x)=$ $v \int K d\left(t^{n} x\right)$ for each $x \in X$. Suppose $P(t)=\sum_{\nu=0}^{n} t^{\nu} x_{\nu}$. Then

$$
\begin{aligned}
T(P(t)) & =\sum_{\nu=0}^{n} T\left(t^{\nu} x_{\nu}\right)=\sum_{\nu=0}^{n} \mu_{\nu}\left(x_{\nu}\right)=\sum_{\nu=0}^{n} v \int_{0}^{1} K d\left(t^{\nu} x_{\nu}\right)+\psi\left(x_{0}\right) \\
& =v \int_{0}^{1} K d\left(\sum_{\nu=1}^{n} t^{\nu} x^{\nu}\right)+\mu\left(x_{\nu}\right) \\
& =v \int_{0}^{1} K d P+\psi(P(0)) .
\end{aligned}
$$

Since the linear transformation on $C$ defined by $v \int_{0}^{1} K d f+\psi(f(0))$ is continuous, and since the polynomials with coefficients in $X$ are continuous, then $T(f)=v \int_{0}^{1} K d f+\psi(f(0))$ for all $f \in C$ and Statement $\mathrm{S}_{1}$ is established.

4. The nonequivalence of the moment problem and convergence preserving. In [4] Kurtz and Tucker stated the following theorem in the setting of linear normed spaces: A Hausdorff method is convergence preserving if and only if it is generated by a moment sequence. However, the proof given succeeded only in establishing that a Hausdorff method generated by a moment sequence is weakly convergence preserving. We now give an example which shows that the stated theorem is incorrect.

Example 4.1. Define $X$ to be $R$, the real numbers and $Y$ to be $l^{\infty}$, the set of all bounded sequences, $\left(\alpha_{1}, \alpha_{2}, \cdots, \alpha_{n}, \cdots\right)$, with sup- 
norm topology. Let $e_{n}$ denote the element of $l^{\infty}$ all of whose terms are $o$ except the $n^{\text {th }}$ term which is 1 . Define the function $f_{m}(\mathrm{t})=$ $m t(1-t)^{m-1}$. Let $t_{1}=1$, and $t_{2}=1 / 2$. There is an integer $m_{3}>2$ such that $f_{m_{3}}\left(1 / m_{3}\right)-f_{m_{3}}(1 / 2)>1 / 2$. Define $t_{3}=1 / m_{3}$. We next define the sequences $\left\{m_{n}\right\}$ and $\left\{t_{n}\right\}$ inductively. Suppose that $t_{i}$ has been defined $i=1, \cdots, \mathrm{n}$. Then, there is an integer $m_{n+1}>1 / t_{n}=m_{n}$ such that $f_{m_{n+1}}\left(1 / m_{n+1}\right)-f_{m_{n+1}}\left(t_{n}\right)>1 / 2$. Define $t_{n+1}=1 / m_{m+1}$. Define the point function $K:[0,1] \rightarrow B[X, Y]=l^{\infty}$ by

$$
K(t)=\left\{\begin{array}{l}
\mathrm{e}_{n} \text { if } t_{n+1}<t \leqq t_{n} \text { for } n=1,2, \cdots \\
0 \text { if } t=0
\end{array}\right.
$$

Observe that $K$ is of bounded semi-variation (has the $\omega$-property [8]) and in fact has semi-variation 2. Furthermore, if $f$ is continuous then $\int_{0}^{1} d k f=\sum_{\nu=2}^{\infty}\left[f\left(t_{\nu}\right)-f\left(t_{\nu-1}\right)\right] e_{\nu}+f(1) e_{1}$. Let $\left(\phi_{n \nu}\right)$ denote the Hausdorff method which is generated by the moment sequence which is defined by $\mu_{n}=\int_{0}^{1} d k t^{n}$. We observe that the sequence $\left\{\dot{\phi}_{n},,_{1}\right\}$ fails to converge. This is seen by the following argument. Suppose $M$ is an integer, then there is an $n>M$ such that $m_{n}\left(t_{M I}\right)\left(1-t_{M I}\right)^{m_{n}-1}$ $<1 / 4$. Then,

$$
\begin{aligned}
& \left\|\dot{\phi}_{m_{M}, 1}-\dot{\phi}_{m_{n}, 1}\right\|=\| \int_{0}^{1} d k\left(m_{M} t_{M I}\left(1-t_{M}\right)^{m_{M}-1}-\int_{0}^{1} d k m_{n} t_{n}\left(1-t_{n}\right)^{n_{n}-1} \|\right. \\
& =\| \sum_{\nu=2}^{\infty}\left[m_{M} t_{\nu}\left(1-t_{\nu}\right)^{m_{M L^{-1}}}-m_{M} t_{\nu-1}\left(1-t_{\nu-1}\right)^{m_{M L}-1}\right]_{\nu} \\
& -\sum_{\nu=2}^{\infty}\left[m_{n}\left(1-t_{\nu}\right)^{m_{n}-1}-m_{n} t_{\nu-1}\left(1-t_{\nu-1}\right)^{m_{n}-1}\right] e_{\nu} \\
& \geqq \|\left[m_{M} t_{M}\left(1-t_{M}\right)^{m_{M}-1}-m_{m} t_{M-1}\left(1-t_{M-1}\right)^{m_{M}-1}\right. \\
& \left.-m_{n} t_{M}\left(1-t_{M}\right)^{m_{n}-1}\right] e_{M} \| \\
& \geqq \frac{1}{4} \text {. }
\end{aligned}
$$

Thus we conclude the sequence fails to be Cauchy. Therefore, Theorem 1.1 implies that $\left(\phi_{n \nu}\right)$ fails to be convergence preserving.

The question of what in addition to being a moment sequence must $\left\{\mu_{n}\right\}$ satisfy in order to generate a convergence preserving method naturally arises. We chose to pursue this question by examining the behavior of the convex set function which generates $\left\{\mu_{n}\right\}$, and we give a condition which is sufficient to guarantee that $\left\{\mu_{n}\right\}$ generates a convergence preserving method.

Definition 4.2. A convex set function $K$ is said to have the Limit Property at 0 if for each $x \in X$ the $\lim _{b \rightarrow 0}[K(a, b]](x)$ exists. If 
for each $x \in X \lim _{b \rightarrow 0}[K(a, b]](x)=\theta_{Y}$ then $K$ is said to have the 0 Limit Property.

THEOREM 4.3. Suppose $\left\{\mu_{n}\right\}$ is generated by a set function $K$ which is of bounded $(p, q)$ convex variation. Then for the Hausdorff method $\left(\dot{\phi}_{n \nu}\right)$ generated by $\left\{\mu_{n}\right\}$ to be convergence preserving, it sufficient that $K$ have the limit property at 0 .

Proof. First observe that (RS) is satisfied by any Hausdorff method $\left(\sum_{\nu=0}^{n} \phi_{n \nu}(x)=\sum_{\nu=0}^{n}\left(\begin{array}{l}n \\ v\end{array}\right) \Delta^{n-\nu} \mu_{\nu}(x)=\mu_{0}(x)\right)$ and that $\left\{\mu_{n}\right\}$ is a moment sequence by 3.2 and hence $(\mathrm{RN})$ is satisfied. Therefore, it is only necessary to establish condition $(\mathrm{C})$. Since $K$ is of bounded $(p, q)$ convex variation, then $\{K(a, b](x):(a, b] \subset(0,1]\}$ is bounded. Therefore, it follows from the Banach-Steinhaus Theorem that there is an $\mathscr{L} \in L[x, y]$ such that $k(a, b] \rightarrow \mathscr{L}$ uniformly on compact subsets of $X$ as $b \rightarrow 0$. Let $K: \mathscr{F} \rightarrow L[X, Y]$, be defined by $K(I)=\mathscr{P}$ for all $I \in \mathcal{F}$. Then $K$ is of bounded $(p, q)$ convex variation as is $K^{*}=$ $K-K$. Suppose $\nu \neq 0$. Choose $x \in X$, and suppose $K$ is $p-q$ related, where $p$ and $q$ are semi-norms in $X$ and $Y$ respectively. Choose $\delta$ such that for $(a, b] \subset(0, \delta], q([K(a, b](x)-L(x))<\varepsilon / 4$. Since $f_{n}(t)=t^{2}(1-t)^{n-\nu} x$ converges uniformly on $[\delta, 1]$ to $\theta_{X}$, then choose $N$ such that $n>N$ implies $\sup _{t} p\left(f_{n}(\mathrm{t})<\varepsilon / 2 W K\right.$. Then, for $n>N$,

$$
\begin{aligned}
q\left(\dot{o}_{n \nu}(x)\right)= & q\left(\left(\begin{array}{l}
n \\
\nu
\end{array}\right) \Delta^{n-\nu} \mu_{\nu}(x)\right) \\
= & q\left(v \int_{0}^{1} K d\left(t^{\nu}(1-t)^{n-\nu} x\right)\right) \\
\leqq & q\left(v \int_{0}^{o} K d\left(t^{\nu}(1-t)^{n-\nu} x\right)\right)+q\left(v \int_{\sigma}^{1} K d\left(t^{\nu}(1-t)^{n-\nu} x\right)\right. \\
\leqq & q\left(v \int_{0}^{\delta} K^{*} d\left(t^{\nu} \cdot(1-t)^{n-\nu} x\right)\right)+q\left(v \int_{0}^{\delta} K d\left(t^{\nu}(1-t)^{n-\nu} x\right)\right) \\
& +W K \sup _{\delta \leqq t \leq 1} q\left(t^{\nu}(1-t)^{\nu} x\right) \\
\leqq & V_{0}^{\delta}\left(t^{\nu}(1-t)^{n-\nu}\right) \sup _{I \subset(0, \delta]}\left[K^{*}(I)\right](x)+\varepsilon / 2 \\
\leqq & 2(\varepsilon / 4)+\varepsilon / 2=\varepsilon .
\end{aligned}
$$

Where in the above $V_{0}^{\delta}\left(t^{\nu}(1-t)^{n-\nu}\right)$ denotes the variation of $f$ over $[0, \delta]$. Hence, $q\left(\phi_{\phi_{\nu}}(x)\right) \rightarrow 0$ as $n \rightarrow \infty$, and it follows that $\dot{\phi}_{n \nu}(x) \rightarrow \theta_{\Gamma}$ as $n \rightarrow \infty$. Suppose $\nu=0$, and $x \in X$. Further, suppose that $K$ is $p-q$ related. Then,

$$
\begin{aligned}
\dot{\phi}_{n, 0}(x) & =\left(v \int_{0}^{1} K^{*} d(1-t)^{n} x\right)+\left(v \int_{0}^{1} K_{\mathscr{x}} d(1-t)^{n} x\right) \\
& =\left(v \int_{0}^{1} K^{*} d(1-t)^{n} x\right)+\int(-x)
\end{aligned}
$$




$$
=\left(w \int_{0}^{1} K^{*} d(1-t)^{n} x\right)-(x)
$$

By an argument similar to the previous one it follows that $v \int_{0}^{1} K^{*} d$ $(1-t)^{n} x$ converges to $\theta_{Y}$ as $n \rightarrow \infty$ and hence that $\phi_{n 0}(x) \rightarrow-\perp(x)$ as $n \rightarrow \infty$. Therefore, $\left(\dot{\phi}_{n \nu}\right)$ satisfies $(C)$ and by 1.1 is a convergence preserving method, and the theorem is established.

In the preceding proof observe that if $\mathscr{C}$ is the identically $\theta_{Y}$ map, then $\phi_{n, \nu}(x) \rightarrow \theta_{Y}$ for all $\nu$. Hence, we have the following corollary to 4.3 .

CoRollary 4.4. Suppose $\left\{\mu_{n}\right\}$ is generated by a set function $K$ which is of bounded $(p, q)$ convex variation. Then for the Hausdorff method generated by $\left\{\mu_{n}\right\}$ to be regular with respect to $\mathscr{L} \in L[X, Y]$ it is sufficient that $\mu_{0}=\subseteq$ and that $K$ have the 0-Limit Property.

5. Concluding remarks. It is not known if the condition that $\left\{\mu_{n}\right\}$ be generated by a $K$ which has the limit property is necessary for the Hausdorff method generated by $\left\{\mu_{n}\right\}$ to be convergence preserving. If $Y$ is a Banach space satisfying the condition that there is a $q>1$ such that $\max (\|x+y\|,\|x-\mathrm{y}\|) \geqq q \min (\|x\|,\|y\|)$, then a $K$ which is of bounded convex variation has the limit property. Hence under this additional condition on $Y$ we conclude that a Hausdorff method is convergence preserving if and only if it is generated by a moment sequence. We note that if $Y$ is a generalized Euclidean space, for example $Y=L_{2}[0,1]$, then the above condition holds with $q=\sqrt{2}$. However, if $Y=L_{1}[0,1]$ or $L_{\infty}[0,1]$ then the above condition does not hold.

6. Addendum to the galley proofs. After this paper was sent to the printer, it was observed that by using inequality (3) on page 400 of (Uniformly Convex Spaces, J. A. Clarkson, Trans. Amer. Math. Soc., 40 (1936), 396-414) one can establish the above condition holds for $L_{p}$ and $l_{p}$ provided $1<p<\infty$. The number $q$ is given by $q=2^{1 / p}$ when $2<p<\infty$, and by $q=2^{1-1 / p}$ when $1<p<2$.

\section{REFERENCES}

1. J. R. Edwards, and S. G. Wayment, A v-integral representation for linear operators on spaces of continuous functions with values in topological vector spaces, Pacific J. Math., 35 (1970), 327-330.

2. - Representations for transformations continuous in the BV Norm. Trans. Amer. Math. Soc., 154 (1971), 251-265.

3. R. K. Goodrich, A Riesz representation theorem and a Hausdorff moment problem Ph. D. Dissertation, University of Utah (1966). 
4. L. C. Kurtz, and D. H. Tucker, Vector-valued summability methods on a linear normed space, Proc. Amer. Math. Soc., 16 (1965), 419-428.

5. M. S. Ramanujan, Generalized Kojima-Toeplitz matrices in certain topological spaces, Math. Ann., 159 (1965), 365-373.

6. - A Hausdorff moment problem for operators in locally convex spaces, Preprint.

7. K. Swong, A representation theory of continuous linear maps, Math. Ann. 155 (1964), 270-291.

8. D. H. Tucker, A representation theorem for a continuous linear transformation on a space of continuous functions, Proc. Amer. Math. Soc., 16 (1965), 946-953.

Received June 3, 1970.

Utah State University 



\section{PACIFIC JOURNAL OF MATHEMATICS}

\section{EDITORS}

H. SAMELSON

Stanford University

Stanford, California 94305

C. R. Hовву

University of Washington

Seattle, Washington 98105
J. DUGUNDJI

Department of Mathematics

University of Southern California

Los Angeles, California 90007

RICHARD ARENS

University of California

Los Angeles, California 90024

\section{ASSOCIATE EDITORS}
E. F. BECKENBACH
B. H. NeumanN
F. WOLF
K. YoSHIDA

\section{SUPPORTING INSTITUTIONS}

UNIVERSITY OF BRITISH COLUMBIA

CALIFORNIA INSTITUTE OF TECHNOLOGY

UNIVERSITY OF CALIFORNIA

MONTANA STATE UNIVERSITY

UNIVERSITY OF NEVADA

NEW MEXICO STATE UNIVERSITY

OREGON STATE UNIVERSITY

UNIVERSITY OF OREGON

OSAKA UNIVERSITY

UNIVERSITY OF SOUTHERN CALIFORNIA
STANFORD UNIVERSITY

UNIVERSITY OF TOKYO

UNIVERSITY OF UTAH

WASHINGTON STATE UNIVERSITY

UNIVERSITY OF WASHINGTON

AMERICAN MATHEMATICAL SOCIETY CHEVRON RESEARCH CORPORATION NAVAL WEAPONS CENTER 


\section{Pacific Journal of Mathematics}

\section{Vol. 38, No. $1 \quad$ March, 1971}

Bruce Alan Barnes, Banach algebras which are ideals in a Banach algebra ..... 1

David W. Boyd, Inequalities for positive integral operators............... 9

Lawrence Gerald Brown, Note on the open mapping theorem .............. 25

Stephen Daniel Comer, Representations by algebras of sections over Boolean

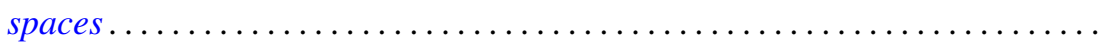

John R. Edwards and Stanley G. Wayment, On the nonequivalence of

conservative Hausdorff methods and Hausdorff moment sequences ........

P. D. T. A. Elliott, On the limiting distribution of additive functions $(\bmod 1) \ldots \ldots$

Mary Rodriguez Embry, Classifying special operators by means of subsets

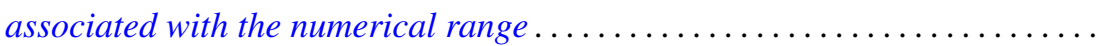

Darald Joe Hartfiel, Counterexamples to a conjecture of G. N. de Oliveira ......

C. Ward Henson, A family of countable homogeneous graphs...............

Satoru Igari and Shigehiko Kuratsubo, A sufficient condition for

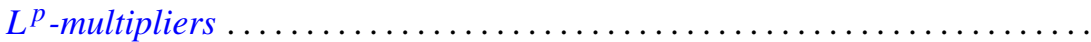

William A. Kirk, Fixed point theorems for nonlinear nonexpansive and

generalized contraction mappings............................

Erwin Kleinfeld, A generalization of commutative and associative rings ...... 95

D. B. Lahiri, Some restricted partition functions. Congruences modulo $11 \ldots \ldots 103$

T. Y. Lin, Homological algebra of stable homotopy ring $\pi *$ of spheres ....... 117

Morris Marden, A representation for the logarithmic derivative of a meromorphic function...........................

John Charles Nichols and James C. Smith, Examples concerning sum properties for metric-dependent dimension functions . .

Asit Baran Raha, On completely Hausdorff-completion of a completely

Hausdorff space.

M. Rajagopalan and Bertram Manuel Schreiber, Ergodic automorphisms and affine transformations of locally compact groups..........

N. V. Rao and Ashoke Kumar Roy, Linear isometries of some function

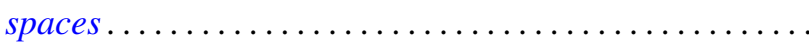

William Francis Reynolds, Blocks and F-class algebras of finite groups

Richard Rochberg, Which linear maps of the disk algebra are multiplicative ...

Gary Sampson, Sharp estimates of convolution transforms in terms of decreasing

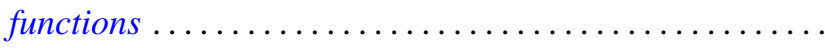

Stephen Scheinberg, Fatou's lemma in normed linear spaces

Ken Shaw, Whittaker constants for entire functions of several complex

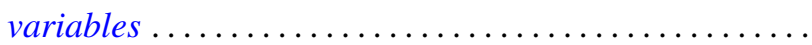

James DeWitt Stein, Two uniform boundedness theorems................ 251

$\mathrm{Li} \mathrm{Pi} \mathrm{Su,} \mathrm{Homomorphisms} \mathrm{of} \mathrm{near-rings} \mathrm{of} \mathrm{continuous} \mathrm{functions} \mathrm{.} \mathrm{.............} 261$

Stephen Willard, Functionally compact spaces, $C$-compact spaces and mappings of minimal Hausdorff spaces....................... 